\title{
Multi-mobile Robot and Avoidance Obstacle to Spatial Mapping in Indoor Environment
}

\author{
Luis Piardi ${ }^{1,2} \mathbb{C}^{\mathrm{a}}$, José Lima $^{1,3} \mathbb{( 1 0}^{\mathrm{b}}$ and André Schneider de Oliveira ${ }^{2} \mathbb{C}^{\mathrm{c}}$ \\ ${ }^{1}$ Research Center in Digitalization and Intelligent Robotics (CeDRI), Instituto Politécnico de Bragança, \\ Campus de Santa Apolónia, 5300-253 Bragança, Portugal \\ ${ }^{2}$ Universidade Técnológica Federal do Paraná - UTFPR, Avenida Sete de Setembro 3165, \\ 80230-901 Curitiba, Paraná, Brazil \\ ${ }^{3}$ INESC, Technology and Science, Porto, Portugal
}

Keywords: $\quad$ Autonomous Mobile Robot, Gas Sources Detection, Avoidance Obstacle, Fuzzy Control.

\begin{abstract}
The advancement of technology and techniques applied to robotics contributes to increasing the quality of life and safety of humanity. One of the most widespread applications of mobile robotics is related to monitoring indoor environments. However, due to factors such as the size of the environment impacting the monitoring response, battery autonomy, and autonomous navigation in environments with unknown obstacles, they are still significant challenges in the diffusion of mobile robotics in these areas. Strategy adopting multiple robots can overcome these challenges. This work presents an approach to use multi-robots in hazardous environments with gas leakage to perform spatial mapping of the gas concentration. Obstacles arranged in the environment are unknown to robots, then a fuzzy control approach is used to avoid the collision. As a result of this paper, spatial mapping of an indoor environment was carried out with multi-robots that reactively react to unknown obstacles considering a point gas leak with Gaussian dispersion.
\end{abstract}

\section{1-INTRODUCTION}

In the past years, academic research has focused on the development of autonomous mobile robots. In this context, several approaches have been explored, for example, robots in the indoor or outdoor environment, using representations with static or dynamic obstacles and total, partial or unknown representations of the environment in which the robot is allocated. Technological advances and the development of new techniques and approaches have disseminated mobile robotics into real problems. Autonomous vehicles are becoming an essential tool in a wide range of environmental applications that include ambient data acquisition, remote sensing, and mapping of the spatial extent of gas leakage (Bayat et al., 2017). The applications of these mobile agents in inspection of industrial plants, search for environmental pollutant sources, explosives, and drugs at airports and harbors are already realities (Braun et al., 2019; Baetz et al., 2009).

\footnotetext{
a (1D) https://orcid.org/0000-0003-1627-8210

b (D) https://orcid.org/0000-0001-7902-1207

c(D) https://orcid.org/0000-0002-8295-366X
}

The present work contributes to the research's tendency in mapping environments contaminated with toxic substances or harmful gases using mobile robots to locate the source of leakage or emission, avoiding the exposure of humans in hazardous environments. For this, a group of four robots will be used, equipped with a sensor and moving collectively towards the indoor source location in a cooperative manner. Obstacles in the environment are unknown, i.e., the robot does not have information about the obstacles' layout and size. The robot control and avoidance obstacle are performed through a controller based on fuzzy logic. During the set of robots navigating the indoor environment with dynamic obstacles, carrying out the acquisition of the gas concentration, the data is analyzed to estimate the leak source position.

The Robot Operating System (ROS) was used to perform tests and validate the proposal of this work. It is a framework that contains a wide range of libraries and tools to develop applications for robots(Martinez and Fernández, 2013). All the development of this work was carried out in a simulation environment, using the Stage Simulator, the $\mathrm{C}++$ software library that simulates multiple mobile robots with a low compu- 
tational cost (Vaughan, 2008).

The remainder of this paper is organized as follows: the next chapter presents a brief review of state of the art considering methods for the spatial mapping of the environment and finding gas leaks, and then a brief presentation of approaches that use mobile robots with fuzzy logic control to avoid obstacles. Chapter 3 presents the system architecture and the simulation environment used in this work. Chapter 4 describes the approach adopted to perform the spatial mapping, namely the robot's trajectories, the fuzzy controllers, and the calculations to estimate the gas's position. In chapter 5 , the results are presented, and finally, in chapter 6 , the conclusions, limitations, and future work are realized.

\section{RELATED WORK}

In this section it will be summarized the briefly review of two topics that this work addresses. The first of these will be indoor gas mapping and detection. The second topic deals with fuzzy logic for the control and avoidance of dynamic and unknown obstacles using mobile robot.

\subsection{Distribution Mapping and Gas Leakage Detection}

There are several ways to get the gas or toxic distribution map in a determined contaminated environment. The most common approach uses stationary sensors, installed in strategic locations, fixed to posts or walls (Kroll et al., 2009). It is a standard approach for monitoring environments that have the risk of gas leakage and can be found even in homes and other buildings (propane and butane based gas detection and smoke detection). Therefore, the gas distribution information is only valid for a limited space around the gas sensor's location. For this approach's efficiency, many sensors are required to cover a relatively large indoor environment efficiently (Fort et al., 2004). Another methodology adopted to carry out the gas distribution mapping is to use specialized and adequately equipped technicians carrying sensors to detect the harmful substances. In this approach, humans are in a contaminated environment to explore and map the substance to locate the emission source.

Considering the limitations of flexibility, robustness and to avoid exposure of humans to high-risk environments, approaches using autonomous mobile robots to perform spatial mapping and leakage focus detection are great alternatives. Therefore, the academic community has been developing robot proto- types to explore contaminated environments, such as (Zakaria et al., 2017; Lilienthal et al., 2009). In this line of research, numerous works study and present in detail a spatial dispersion model of gases, as can be analyzed in (Kowadlo and Russell, 2008; Lilienthal and Duckett, 2004; Loutfi et al., 2009; Lilienthal et al., 2006).

The need for the use of mobile robots occurs mainly when the gas source is composed of toxic or explosive gases, need for flexibility or replacement of sensors, when the gas source occurs in an inaccessible location, or when a continuous verification of the environment is necessary (Gongora et al., 2017). Approaches that use a mobile robot with a gas sensor attached are found in the literature (Piardi et al., 2017; Braun et al., 2019). However, these have limitations for large environments due to battery limitations and delay in the response of gas detection given the size of the environment. Strategies based on genetic algorithms seek to optimize the robot's route to perform more efficient monitoring, however for dynamic environments, changes in the environment demand time to re-calculate the ideal route (Piardi et al., 2018). Multirobot approaches for monitoring can map larger environments and be more responsive to detecting gas leakage sources.

\subsection{Fuzzy Logic Control}

Mobile robots have a wide range of use and can be controlled using telemetry or semi-autonomous and autonomous approach. For a robot to be fully autonomous and therefore independent of human operators or users' decisions, controllers that operate at their motors' speeds are required, usually feedforward controller or feedback controller. In (Pandey et al., 2017) it is possible to obtain information of different techniques used to equip robots of autonomy, such as neural networks, genetic algorithms, and fuzzy controllers. Sensors with a high amount of environment information have been widely used to avoid collision with obstacles, as presented in (Morais et al., 2017), which uses an RGB-D sensor (e.g kinect or intel real sense) applying Artificial Potential Field to avoid nearby obstacles. However, approaches based on RGB-D sensors demand a high computational power to process all environment around the robot, identify obstacles and execute a path free of collision.

In particular, for this work, the fuzzy control approach will be used, which was introduced by Zadeh (Zadeh, 1975). It is widely adopted to control the speed applied to the robot's wheels to control threedimensional coordinates involved i.e. $[x, y, \theta]$. Fuzzy logic is especially useful for robot controllers, and re- 
searchers present different approaches to control the robot with fuzzy logic (Vinogradov et al., 2019; Avelar et al., 2020; Mousavi, 2015). The approach proposed in this work uses two fuzzy controls to operate the wells robot speed, one without the presence of obstacles whose input is the position of the robot obtained from optometry and the second is active when an obstacle is detected near to the robot, whose input is the distance and direction of the obstacle.

\section{SETUP AND SYSTEM ARCHITECTURE}

For the present work, the ROS was used as the framework to control four mobile robots Pioneer model P3-DX with a differential drive. Each robot was equipped with Hokuyo laser scanner model URG04LX to obtain information about the environment and unknown obstacles. The test and validation environment was developed in the Stage simulator. This software presents a realistic model of the 3D environment, representing the dynamic constraints in real applications. This environment has 400 squared meters where it is distributed different unknown obstacles by the robots. Figure 1a shows the simulation environment where the blocks in black are walls, in blues are obstacles that can be moved and in red arranged in the corners of the environment are the robots. Figure $1 \mathrm{~b}$ shows the proposed system architecture describing the data flow.

The data obtained from the simulator (pose from dead reckoning, gas data and laser scan data) are processed in a project developed in python. The multirobots are controlled to perform a defined trajectory, aiming the exploration of the environment and acquisition of gas concentration data. At the end of the trajectory, the stored gas data are plotted showing the gas concentration at the points visited by the robots. As the objective of this work is not to propose a new method for gas dispersion, the adopted model is generic and simplified (Kowadlo and Russell, 2008), not taking into account wind intensity and orientation, temperature and chemical characteristics of gas particles. Equation (1) presents the model of gas dispersion in the adopted environment, whose behavior is a Gaussian function.

$$
Z(x, y)=A \cdot e^{-\frac{\left(x-x_{0}\right)^{2}+\left(y-y_{0}\right)^{2}}{2 \cdot \sigma^{2}}}
$$

The variable $Z$ indicates the concentration of gas, while $(x ; y)$ the position of the robot. The variable $A$ represents the height of the curve's peak. The leakage position is described as $\left(x_{0} ; y_{0}\right)$ and $\sigma$ (the standard

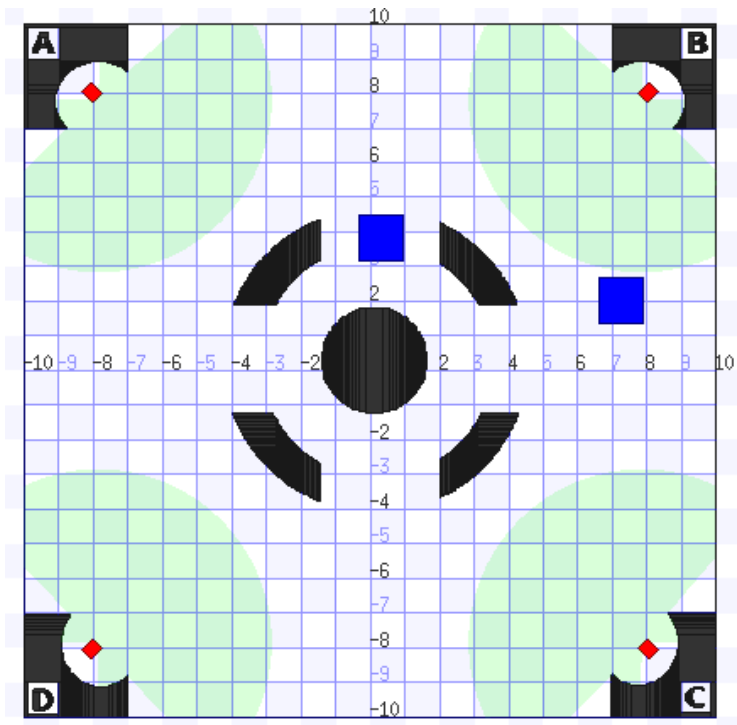

(a) Stage simulation environment.

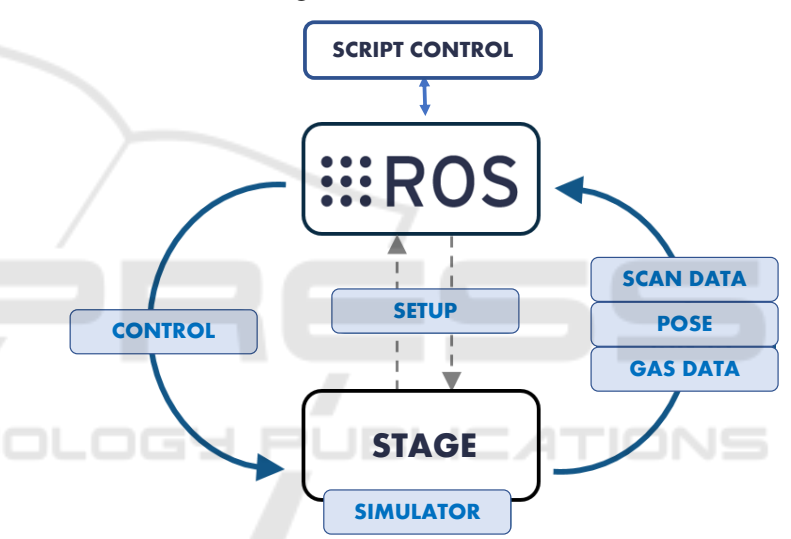

(b) System Architecture.

Figure 1: (a) Simulation environment and (b) architecture proposed.

deviation) the parameter to control the width of the "belt".

\section{PROPOSED APPROACH}

In this section it will be presented the approach developed to perform a spatial mapping of gas distribution in an indoor environment. Initially, the robot's trajectory will be described, covering a region enough to map the environment. Then, the approach of the fuzzy controller to follow the path and avoid the unknown obstacles is presented and finally a mathematical model to estimate the position of the source of gas emission. 


\subsection{Robot's Trajectory}

Considering that performing a complete coverage of the environment with a large size may require a significant amount of time and is limited to battery capacity of the mobile robots. The proposed trajectory aims at an alternative to perform the data acquisition for spatial mapping. The simulation environment has the dimension of -10 to 10 meters in both the $X$ coordinate and the $Y$ coordinate. Table 1 shows the initial, intermediate and final position for each robot. With this trajectory, the robots tend to perform a diagonal crossing in the environment and then move clockwise direction, ending the trajectory, illustrated by the Figure 2 .

Table 1: Trajectory waypoints $(x ; y)$ for each robot.

\begin{tabular}{cccc}
\hline Robot & Initial Position & Intermediate position & End position \\
\hline A & $(-8 ; 8)$ & $(7 ;-7)$ & $(-6 ;-6)$ \\
B & $(8 ; 8)$ & $(-7 ;-7)$ & $(-6 ; 6)$ \\
C & $(8 ;-8)$ & $(-7 ; 7)$ & $(6 ; 6)$ \\
D & $(-8 ;-8)$ & $(7 ; 7)$ & $(6 ;-6)$ \\
\hline
\end{tabular}

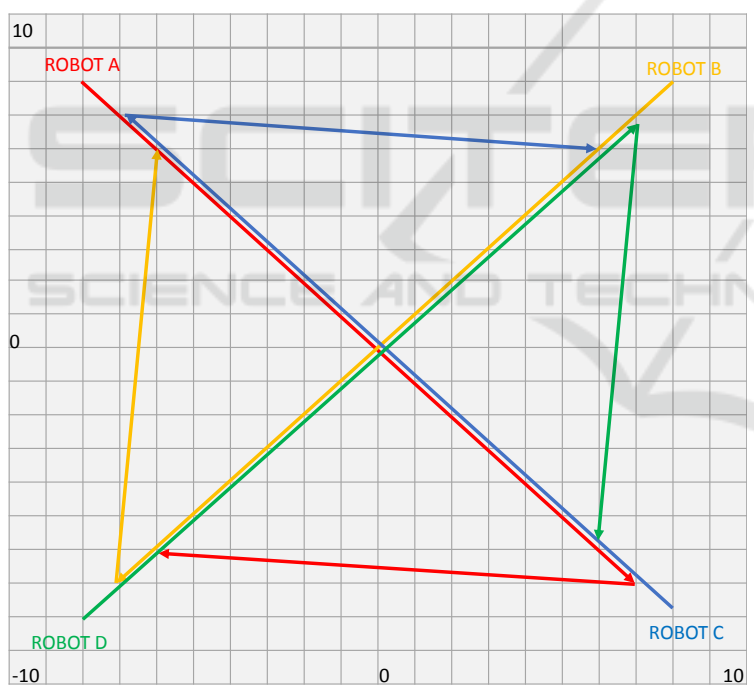

Figure 2: Trajectories for robots to explore the environment.

It is worth mentioning that deviations from the initial trajectory can occur due to the existence of unknown obstacles. With this arrangement, all portions of the environment will have sample gas concentration data.

\subsection{Fuzzy Logic Control}

The goal of fuzzy logic control is to generate linear and angular velocity, which will be converted through ROS to velocities for both the mobile robot's right and left motor. To the robots navigate and find their tar- get while avoiding obstacles, an approach using two fuzzy controllers has been developed. This approach was inspired by (Faisal et al., 2013), changing the use of a sonar sensor to a LiDAR, making it possible to extract more information from the environment and, consequently, completely modify fuzzy memberships and rules. The first one is free path fuzzy control (FPFC). The second one logic is free obstacle fuzzy control (FOFC), triggered when the scan laser sensor detects an obstacle inside a radius of 1 meter from the robot. Figure 3 illustrates the fuzzy controller of FPFC and FOFC through a flowchart.

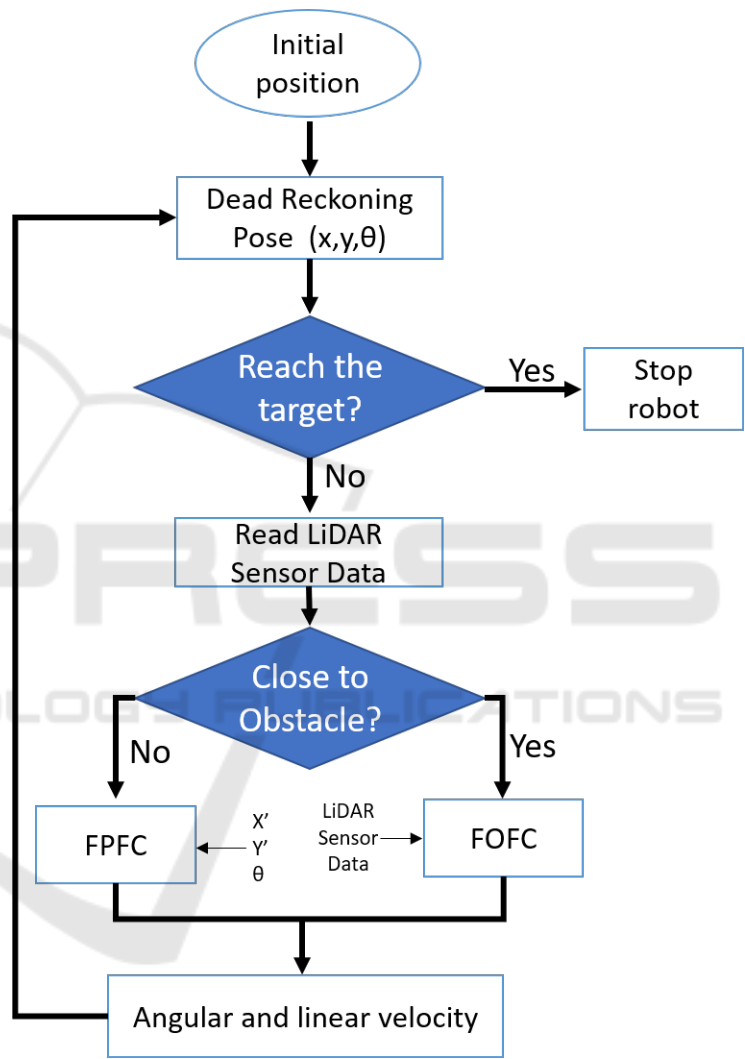

Figure 3: Flowchart of fuzzy logics for robot control.

\subsubsection{Free Path Fuzzy Control}

FPFC is proposed to move the robot to target points smoothly. The inputs of FPFC are the distance from the robot to the target and the angle between the robot and the target (error angle) as shown in Figure 4.

The outputs of FPFC are linear and angular speed of the mobile robot. FPFC is implemented with 3 membership functions for each input as illustrated in Figure 5 and Figure 6. The fuzzy control rules for these two inputs and two outputs for control the linear and angular velocity are shown in Table 2 .

The velocities (linear and angular) of the mobile 


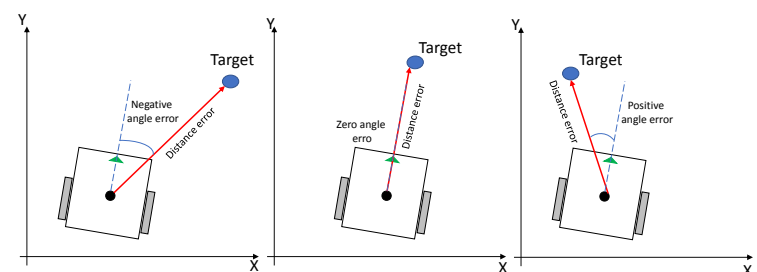

Figure 4: Cases related to distance and negative, zero and positive angles for FPFC.

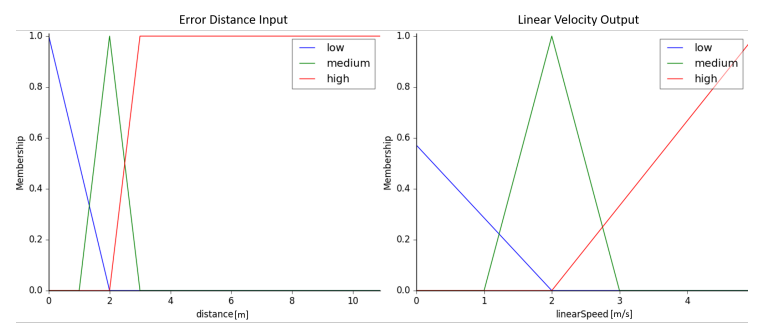

Figure 5: Input membership functions for the distance error and membership functions for the linear velocity.

robots are calculated using the defuzzification step. Note that in this work, the centroid defuzzification technique has been adopted.

\subsubsection{Free Obstacle Fuzzy Control}

The FOFC enters the scene in place of FPFC when the laser scan detects some obstacle within 1 meter of the distance around the robot $\left(0\right.$ to $\left.180^{\circ}\right)$. In this control methodology, the robot does not collide with unknown obstacles in the environment, generating linear and angular velocities so that the robot's trajectory is free of collisions. Figure 7 shows the used method on how the inputs to the fuzzy logic are obtained.

This logic consists of two inputs and two outputs. The first input represents the shortest distance between any obstacle and the robot (yellow line in Figure 7), while the second input indicates the angle of the line representing this shortest distance from the robot and the obstacle. The outputs of FOFC are the linear and angular velocities. FOFC is implemented with three membership functions for distance (input and output) illustrated in Figure 8. For the angular

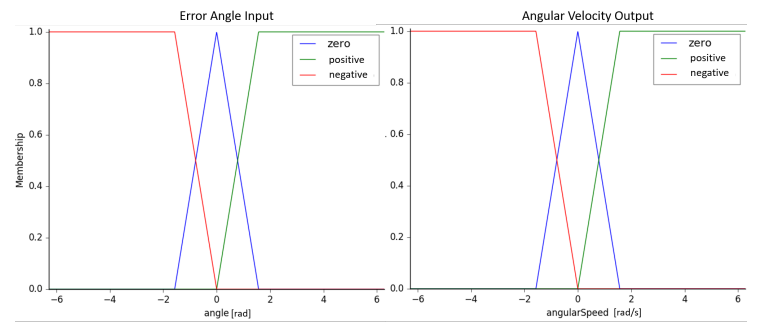

Figure 6: Membership functions for the angle error and membership functions for the angular velocity.
Table 2: Fuzzy rules of the linear and angular velocity of the robot in FPFC.

\begin{tabular}{cccc}
\hline \multicolumn{2}{c}{ Input } & \multicolumn{2}{c}{ Output } \\
\hline Distance Error & Angle Error & Linear Velocity & Angular Velocity \\
\hline low & zero & low & zero \\
low & positive & low & positive \\
low & negative & low & negative \\
medium & zero & medium & zero \\
medium & positive & medium & positive \\
medium & negative & medium & negative \\
high & zero & high & zero \\
high & positive & high & positive \\
high & negative & high & negative \\
\hline
\end{tabular}

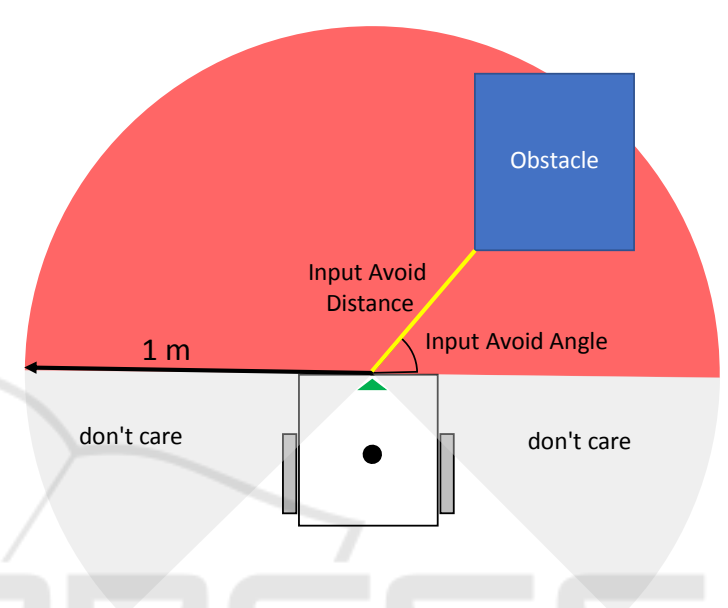

Figure 7: Illustration for the input values for the FOFC.

input, five membership functions are used, while for the output, three functions are used illustrated in Figure 9. The rule of FOFC for these two inputs and two outputs for control the linear and angular velocity avoiding obstacle is shown in Table 3.

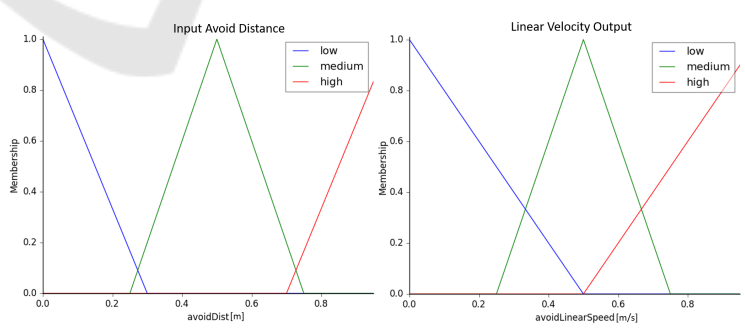

Figure 8: Input membership functions for the distance to avoid obstacle and membership functions for the linear velocity.

\subsection{Algorithm to Find the Gas Source}

Considering equation (1) which simplifies the behavior of the gas dispersion in a Gaussian function and the fact that the robot, while following its trajectory, records position data and its gas concentration, mathematically it is possible to estimate the position of the 


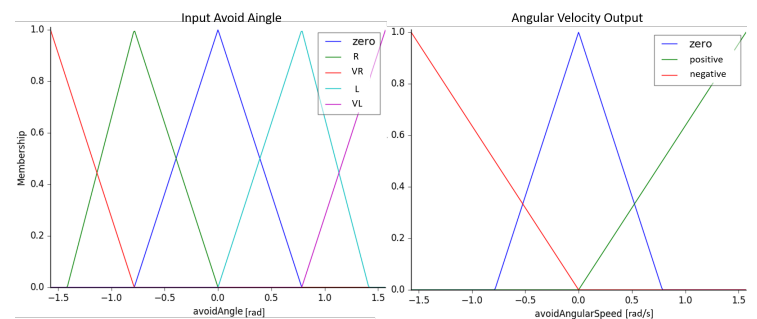

Figure 9: Membership functions for the angle to avoid obstacle and membership functions for the angular velocity.

source with the stored gas concentration data from each robots. After completing the entire trajectory, each robot will contribute with the highest gas concentration obtained $Z$ and the position $(x, y)$ of this measurement.

Table 3: Fuzzy rules of the linear and angular velocity of the robot in FOCF.

\begin{tabular}{cccc}
\hline \multicolumn{2}{c}{ Input } & \multicolumn{3}{c}{ Output } \\
\hline Distance & Angle & Linear Velocity & Angular Velocity \\
\hline low & zero & medium & negative \\
low & $\mathrm{E}$ & medium & negative \\
low & $\mathrm{D}$ & medium & positive \\
low & $\mathrm{ME}$ & medium & zero \\
medium & zero & medium & zero \\
medium & $\mathrm{E}$ & medium & negative \\
medium & $\mathrm{D}$ & medium & negative \\
medium & $\mathrm{ME}$ & medium & positive \\
medium & $\mathrm{MD}$ & medium & zero \\
high & zero & medium & zero \\
high & $\mathrm{E}$ & medium & negative \\
high & $\mathrm{D}$ & medium & zero \\
high & $\mathrm{ME}$ & medium & zero \\
high & $\mathrm{MD}$ & medium & zero \\
& & & zero \\
\hline
\end{tabular}

Then, it is possible to use the Linear Least Squares estimation to find the $\left(x_{0}, y_{0}\right)$ gas emission origin, with $Z$ concentration measure.

$$
\ln Z=\ln A+B\left(x^{2}-2 x x_{0}+x_{0}^{2}+y^{2}-2 y y_{0}+y_{0}^{2}\right)
$$

From equation 2, where $B=-\frac{1}{2 \sigma^{2}}$, and rearranging the parcels, results on the equation (3)

$\ln Z=B\left(x^{2}+y^{2}\right)-2 B x_{0} x-2 B y_{0} y+\ln A+B\left(x_{0}^{2}+y_{0}^{2}\right)$

The model for the Linear Least Square is found in equation (4).

$$
w=\alpha_{1} u_{1}+\alpha_{2} u_{2}+\alpha_{3} u_{3}+\alpha_{4} u_{4}
$$

The Linear Least Square variables represent the following system values:

$$
\begin{aligned}
& \alpha_{1}=B \\
& \alpha_{2}=-2 B x_{0} \\
& \alpha_{3}=-2 B y_{0} \\
& \alpha_{4}=\ln A+B\left(x_{0}^{2}+y_{0}^{2}\right) \\
& w=\ln Z \\
& u_{1}=x^{2}+y^{2} \\
& u_{2}=x \\
& u_{3}=y \\
& u_{4}=1 \\
& \alpha=\left(U^{T} . U\right)^{-1} \cdot U^{T} . W \\
& \alpha=\left[\begin{array}{llll}
\alpha_{1} & \alpha_{2} & \alpha_{3} & \alpha_{4}
\end{array}\right]
\end{aligned}
$$

The matrices in equation (5) are defined in equations (6), (7) and (8) assuming $n$ measures, where $n$ indicates the number of robots, and in this case $n=4$. After the estimation the gas emission origin $\left(x_{0}, y_{0}\right)$ can be found by the expressions in equation (9).

$$
B=\alpha_{1}, x_{0}=-\frac{\alpha_{2}}{2 B}, y_{0}=-\frac{\alpha_{3}}{2 B}
$$

Therefore, after applying the maximum values of gas concentration obtained by each robot during the execution of its defined trajectory, in the Linear Least Square model to obtain the values of $\alpha_{1}$ a1, $\alpha_{2}, \alpha_{4}$, and $\alpha_{4}$, and substitute these variables in equation 9 , the source of the gas leak $x_{0}$ and $y_{0}$ is estimated.

\section{RESULTS}

In order to present the results of this work, the approach described above with four robots was performed in the STAGE simulator, a multi mobile robot simulator contained in ROS, to obtain samples of gas concentration data in an environment of 20 X 20 meters. Figure 10 shows the gas distribution in the environment according to equation 1 with the gas source 
located at the position $x_{0}=-2$ and $y_{0}=3$.It is possible to observe the Gaussian behavior of the gas concentration throughout the environment, which decays exponentially as it moves away from the source of the gas leak in any direction.

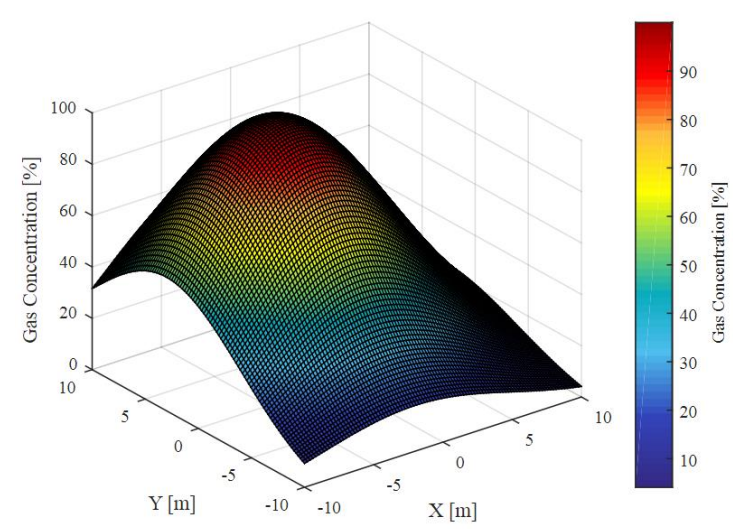

Figure 10: Gaussian distribution of gas in an indoor environment, source of gas located at position $x_{0}=-2$ and $y_{0}=$ 3.

Figure 11 shows the gas samples collected by the robots during their path as described in section 4.1. The multiple mobile robots carry out the trajectory simultaneously. Even robots do not perform all the scanning of the environment, it is possible to observe that at some moment of the defined path, some robot will pass near to a point with a high concentration of gas (whatever it is), which is enough to estimate the position of the gas source.

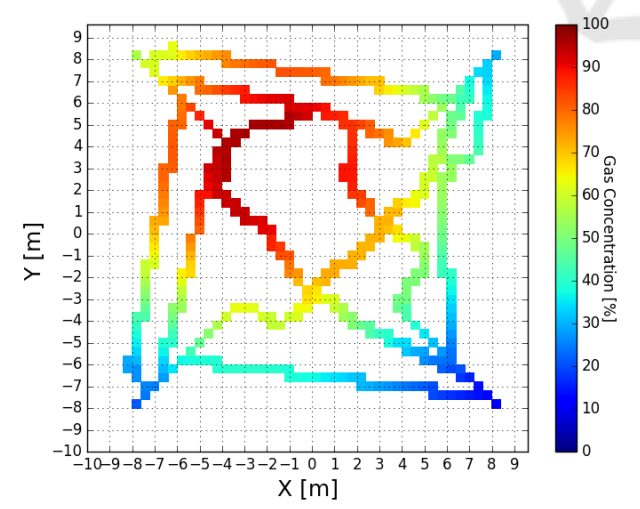

Figure 11: Robots detection of gas in the indoor environment, source of gas located at position $x_{0}=-2$ and $y_{0}=3$.

Figure 12 divides the trajectory into 6 scenarios. Scenario 1 shows the start point of robots A, B, C, and $\mathrm{D}$ with the handle sensors (circle in green) according to Table 1. They start at the intermediate point according to the FPFC algorithm. In scenario 2, it is possible to identify that due to the presence of un- known obstacles detected by the LiDAR sensor, the FOFC controller is active, and there were no collisions, and the robots start to bypass the obstacles in the region of nearly 1 meter of distance. Scenario 3 and 4 is a region that alternates between the two configurations (FPFC or FOFC) according to the robot's position. In scenario 5 , all robots are already at the intermediate point towards the endpoint (scenario 6), predominantly controlled by the FPFC rules.

Table 4: Data obtained by the robots to estimate the position of the gas leak.

\begin{tabular}{lccc}
\hline & $\mathrm{x}[\mathrm{m}]$ & $\mathrm{y}[\mathrm{m}]$ & Gas Conc. $(\mathrm{Z})[\%]$ \\
\hline Robot A & 0.939 & -1.731 & 72.861 \\
Robot B & -1.430 & 5.828 & 91.858 \\
Robot C & -3.847 & 3.399 & 96.421 \\
Robot D & -2.32 & 4.84 & 96.490 \\
\hline
\end{tabular}

The complete trajectory executed by the robots to reach the target avoiding obstacle can be seeing in the complementary video available at: https://tinyurl. com/tpysdbf5.

The position $(x, y)$ where each robot detected the highest gas concentration, as well as the percentage of this concentration is described in Table 4. After applying the logarithmic calculation to the $Z$ concentration values and obtaining the $w$ values of each robot to construct the $W$ matrix (equation 7) and the calculations with $(x, y)$ positions to obtain the variables $u_{1}$, $u_{2}, u_{3}$ and $u_{4}$, doing this for each robot and then obtaining the $U$ matrix (equation 8 ) to result in the values of $\alpha$ (equation 5 and 6 ) the system of equation is then solved (equation 9), resulting in the position of the gas leak source $x_{0}=-2$ and $y_{0}=3$.

\section{CONCLUSIONS AND FUTURE WORKS}

In this work, a multi-robot system was developed in ROS using STAGE simulator, to carry out the scanning in high risk indoor environments, which objective is to obtain a spatial mapping of the gas concentration. The fuzzy system proposed for the work, namely FPFC and FOFC based in the Lidar sensor distance data, proved to be efficient, since the robots do not collide with unknown obstacles and managing to cross tight spaces between two distinct obstacles. Considering the simplification of gas dispersion in the environment, it was possible to simulate its distribution and, applying the Linear Least Squares model presented, estimate the position of the gas leak source. 

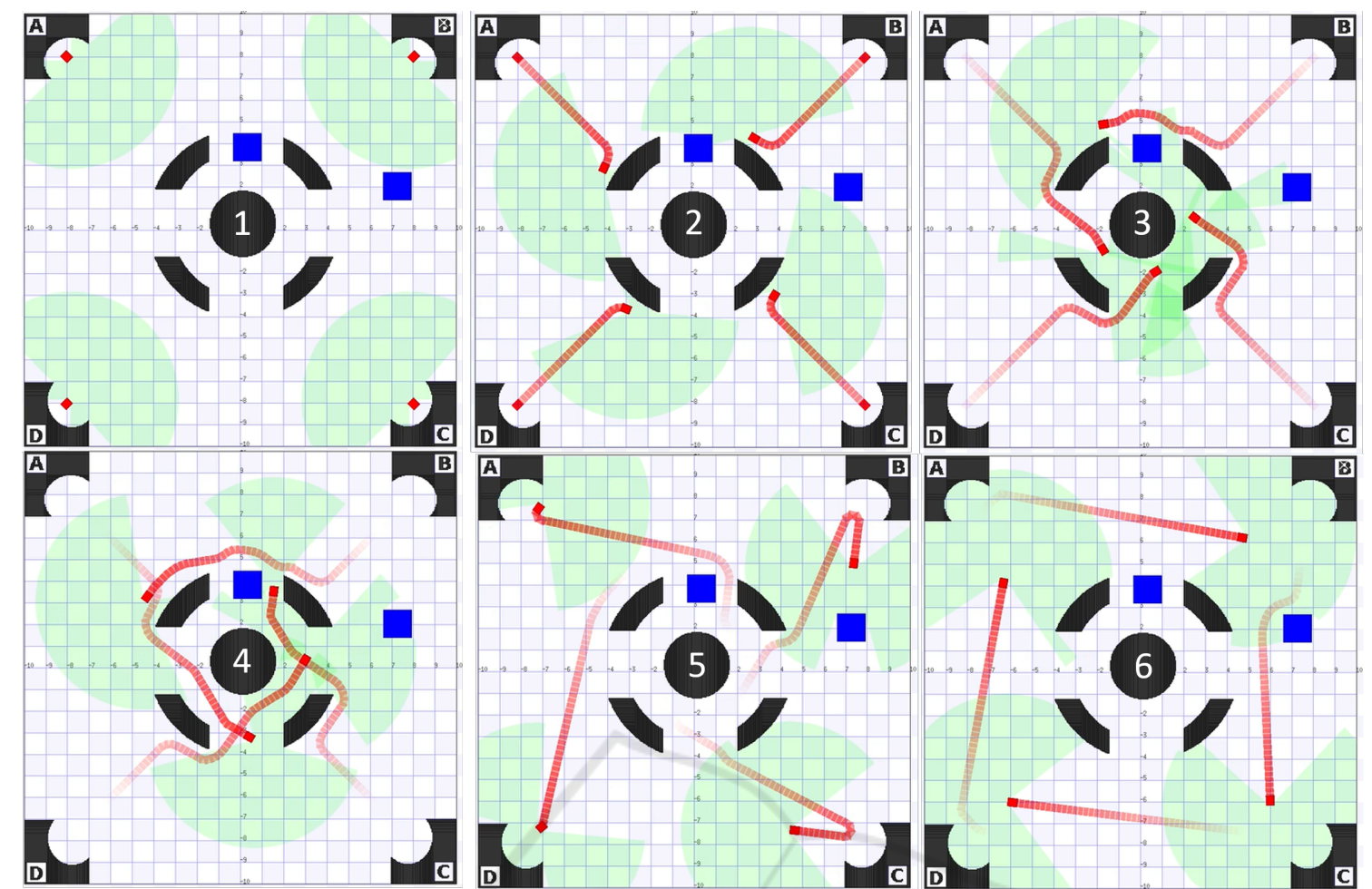

Figure 12: FPFC and FOFC algorithm to multi robot free collisions navigation in the environment with unknown obstacles.

The known limitations of this work will be explored in future works, such as interference and noise in the use of several laser sensors with similar characteristics and frequency bands and calibration and delay correction in multi-robot systems. More complex gas distribution models will also be implemented in future works, considering the effect of wind, temperature, and particle characteristics, adapting the gas leak source's pose estimation technique. Finally, as the interaction between robots to optimize space explored and monitored in the indoor environment, task allocation policies and data exchange regarding gas concentrations in the scanned area by each robot.

\section{ACKNOWLEDGMENT}

This work has been supported by FCT- Fundação para a Ciência e Tecnologia within the Project Scope: UIDB/05757/2020. Additionally, this work was supported in part by the National Counsel of Technological and Scientific Development of Brazil (CNPq), in part by the Coordination for the Improvement of Higher Level People (CAPES).

\section{REFERENCES}

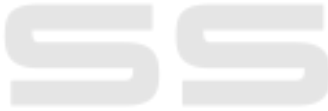

Avelar, E., Castillo, O., and Soria, J. (2020). Fuzzy logic controller with fuzzylab python library and the robot operating system for autonomous mobile robot navigation. Journal of Automation Mobile Robotics and Intelligent Systems, 14.

Baetz, W., Kroll, A., and Bonow, G. (2009). Mobile robots with active ir-optical sensing for remote gas detection and source localization. In 2009 IEEE International Conference on Robotics and Automation, pages 27732778. IEEE.

Bayat, B., Crasta, N., Crespi, A., Pascoal, A. M., and Ijspeert, A. (2017). Environmental monitoring using autonomous vehicles: a survey of recent searching techniques. Current opinion in biotechnology, 45:7684.

Braun, J., Piardi, L., Brito, T., Lima, J., Pereira, A., Costa, P., and Nakano, A. (2019). Indoor environment monitoring in search of gas leakage by mobile robot. In Iberian Robotics conference, pages 339-350. Springer.

Faisal, M., Hedjar, R., Al Sulaiman, M., and Al-Mutib, K. (2013). Fuzzy logic navigation and obstacle avoidance by a mobile robot in an unknown dynamic environment. International Journal of Advanced Robotic Systems, 10(1):37.

Fort, A., Serrano-Santos, M., Spinicci, R., Ulivieri, N., and Vignoli, V. (2004). Electronic noses based on metal oxide gas sensors: the problem of selectivity 
enhancement. In Proceedings of the 21st IEEE Instrumentation and Measurement Technology Conference (IEEE Cat. No. 04CH37510), volume 1, pages 599604. IEEE.

Gongora, A., Monroy, J., and Gonzalez-Jimenez, J. (2017). Gas source localization strategies for teleoperated mobile robots. an experimental analysis. In 2017 European Conference on Mobile Robots (ECMR), pages 16. IEEE.

Kowadlo, G. and Russell, R. A. (2008). Robot odor localization: a taxonomy and survey. The International Journal of Robotics Research, 27(8):869-894.

Kroll, A., Baetz, W., and Peretzki, D. (2009). On autonomous detection of pressured air and gas leaks using passive ir-thermography for mobile robot application. In Robotics and Automation, 2009. ICRA'09. IEEE International Conference on, pages 921-926. IEEE.

Lilienthal, A. and Duckett, T. (2004). Building gas concentration gridmaps with a mobile robot. Robotics and Autonomous Systems, 48(1):3-16.

Lilienthal, A., Loutfi, A., and Duckett, T. (2006). Airborne chemical sensing with mobile robots. Sensors, 6(11):1616-1678.

Lilienthal, A. J., Reggente, M., Trincavelli, M., Blanco, J. L., and Gonzalez, J. (2009). A statistical approach to gas distribution modelling with mobile robots-the kernel dm+ v algorithm. In 2009 IEEE/RSJ International Conference on Intelligent Robots and Systems, pages 570-576. IEEE

Loutfi, A., Coradeschi, S., Lilienthal, A. J., and Gonzalez, J. (2009). Gas distribution mapping of multiple odour sources using a mobile robot. Robotica, 27(2):311319.

Martinez, A. and Fernández, E. (2013). Learning ROS for robotics programming. Packt Publishing Ltd.

Morais, C., do Nascimento, T. P., Brito, A. V., and Basso, G. (2017). A 3d anti-collision system based on artificial potential field method for a mobile robot. In ICAART (1), pages 308-313.

Mousavi, M. A. (2015). A fast fuzzy path tracking controller for mobile robots. In 2015 3rd RSI International Conference on Robotics and Mechatronics (ICROM), pages 675-680. IEEE.

Pandey, A., Pandey, S., and Parhi, D. (2017). Mobile robot navigation and obstacle avoidance techniques: A review. Int Rob Auto J, 2(3):00022.

Piardi, L., Lima, J., Costa, P., and Brito, T. (2017). Development of a dynamic path for a toxic substances mapping mobile robot in industry environment. In Iberian Robotics conference, pages 655-667. Springer.

Piardi, L., Lima, J., Pereira, A. I., and Costa, P. (2018). Path planning optimization method based on genetic algorithm for mapping toxic environment. In International Conference on Bioinspired Methods and Their Applications, pages 223-233. Springer.

Vaughan, R. (2008). Massively multi-robot simulation in stage. Swarm intelligence, 2(2-4):189-208.

Vinogradov, A., Terentev, A., Kochetkov, M., and Petrov, V. (2019). Model of fuzzy regulator of mobile robot motion control system. In 2019 IEEE Conference of Rus- sian Young Researchers in Electrical and Electronic Engineering (EIConRus), pages 2109-2112. IEEE.

Zadeh, L. A. (1975). The concept of a linguistic variable and its application to approximate reasoning-i. Information sciences, 8(3):199-249.

Zakaria, A. H., Mustafah, Y. M., Abdullah, J., Khair, N., and Abdullah, T. (2017). Development of autonomous radiation mapping robot. Procedia Computer Science, 105:81-86. 